\title{
BMJ Open Comorbidity in adults with traumatic brain injury and all-cause mortality: a systematic review
}

\author{
Chen Xiong, ${ }^{1,2,3}$ Sara Hanafy, ${ }^{1,2,3}$ Vincy Chan, ${ }^{2,3}$ Zheng Jing Hu, ${ }^{2,4}$ Mitchell Sutton, ${ }^{2}$ \\ Michael Escobar, ${ }^{4}$ Angela Colantonio, ${ }^{1,2,3,5}$ Tatyana Mollayeva (iD) ${ }^{2,3}$
}

To cite: Xiong C, Hanafy S, Chan V, et al. Comorbidity in adults with traumatic brain injury and all-cause mortality: a systematic review. BMJ Open 2019;9:e029072. doi:10.1136/ bmjopen-2019-029072

- Prepublication history and additional material for this paper are available online. To view these files, please visit the journal online (http://dx.doi org/10.1136/bmjopen-2019029072).

Received 10 January 2019 Revised 30 April 2019 Accepted 22 July 2019

Check for updates

(c) Author(s) (or their employer(s)) 2019. Re-use permitted under CC BY-NC. No commercial re-use. See rights and permissions. Published by BMJ.

${ }^{1}$ Rehabilitation Sciences Institute, Faculty of Medicine, University of Toronto, Toronto, Ontario, Canada

${ }^{2}$ KITE-Toronto Rehab-University Health Network, Toronto,

Ontario, Canada

${ }^{3}$ Acquired Brain Injury Research Lab, University of Toronto, Toronto, Ontario, Canada ${ }^{4}$ Dalla Lana School of Public Health, University of Toronto, Toronto, Ontario, Canada

${ }^{5}$ Department of Occupational

Science and Occupational

Therapy, University of Toronto, Toronto, Ontario, Canada

Correspondence to

Chen Xiong;

chen.xiong@mail.utoronto.ca

\section{ABSTRACT}

Objectives Comorbidity in traumatic brain injury (TBI) has been recognised to alter the clinical course of patients and influence short-term and long-term outcomes. We synthesised the evidence on the effects of different comorbid conditions on early and late mortality postTBI in order to (1) examine the relationship between comorbid condition(s) and all-cause mortality in TBI and (2) determine the influence of sociodemographic and clinical characteristics of patients with a TBI at baseline on allcause mortality.

Design Systematic review.

Data sources Medline, Central, Embase, PsycINF0 and bibliographies of identified articles were searched from May 1997 to January 2019.

Eligibility criteria for selecting studies Included studies met the following criteria: (1) focused on comorbidity as it related to our outcome of interest in adults (ie, $\geq 18$ years of age) diagnosed with a TBI; (2) comorbidity was detected by any means excluding self-report; (3) reported the proportion of participants without comorbidity and (4) followed participants for any period of time.

Data extraction and synthesis Two independent reviewers extracted the data and assessed risk of bias using the Quality in Prognosis Studies tool. Data were synthesised through tabulation and qualitative description. Results A total of 27 cohort studies were included. Among the wide range of individual comorbid conditions studied, only low blood pressure was a consistent predictors of post-TBI mortality. Other consistent predictors were traditional sociodemographic risk factors. Higher comorbidity scale, scores and the number of comorbid conditions were not consistently associated with post-TBI mortality.

Conclusions Given the high number of comorbid conditions that were examined by the single studies, research is required to further substantiate the evidence and address conflicting findings. Finally, an enhanced set of comorbidity measures that are suited for the TBI population will allow for better risk stratification to guide TBI management and treatment.

PROSPERO registration number CRD42017070033

\section{INTRODUCTION}

A traumatic brain injury (TBI), defined as 'an alteration in brain function or other evidence of brain pathology, caused by an external

\section{Strengths and limitations of this study}

This systematic review is the first systematic review that investigated the relationship between comorbidity, sociodemographic and clinical characteristics and all-cause mortality in populations with traumatic brain injury (TBI).

- The Quality in Prognosis Studies tool was used to evaluate the quality of the available evidence.

- We acknowledge heterogeneity in the included studies, which demonstrate a great deal of variations in the populations studied, forms and types of comorbidities examined and the timing of mortality outcome. As such, meta-analyses were not performed.

- Further studies on the effect of comorbidity on mortality throughout the life course in patients with TBI are necessary.

force', ${ }^{1}$ is a major public health concern and a leading cause of death and disability across the world. ${ }^{2}$ Globally, TBI is among the top three neurological conditions accounting for disability. ${ }^{2}$ Specifically, approximately 50-60 million new TBI cases are estimated to occur annually. ${ }^{2}$ Over 200 per 100000 individuals with TBI are admitted to European hospitals each year, with an average in-hospital case fatality rate of $3 \%$; in the USA, the average rate is $6.2 \%$, and estimates indicate that $1 \%-2 \%$ of the population live with disability caused by TBI. ${ }^{3}$ Among those who survive, injury-related physical and cognitive impairments are often lifelong. In addition to experiencing disabilities, the challenges of adjusting to changing roles and responsibilities postinjury may result in exacerbation of pre-existing conditions or expedition of the development of new disorders and clinical clusters (ie, comorbidities), including but not limited to anxiety, mood, pain and cognitive disorders, thereby increasing the associated direct and indirect medical costs. ${ }^{4-6}$

Comorbidity in TBI has long been recognised to alter the clinical course of patients by affecting selection of both early 
and long-term healthcare services postinjury, and hence influencing short-term and long-term outcomes ${ }^{7-9}$ Recent timely initiatives have recommended that an assessment of comorbidities be included among the TBI population, which is extremely important, as the presence of comorbidity or multiple comorbidities in patients with TBI is common and has shown to be associated with all-cause mortality. ${ }^{10-13}$ In addition to comorbidities, advanced age and male sex have also been found to be associated with elevated TBI-related mortality rates. ${ }^{14}{ }^{15}$ While a number of previous research studies have highlighted the types and number of comorbidities among patients with TBI, ${ }^{10}$ there remains a paucity of evidence synthesis on the effects of different comorbid conditions on early and late mortality post-TBI, taking into account distribution of comorbidity across the age span and among sexes.

To address the highlighted research gaps, the primary objective of this systematic review was to: (1) examine the relationship between comorbid condition(s) and allcause mortality in TBI and (2) determine the influence of sociodemographic and clinical characteristics of patients with a TBI at baseline on the development of adverse or beneficial outcome (ie, mortality or survivorship) across time.

\section{METHODS}

The systematic review was conducted based on a previously peer-reviewed protocol registered with the International Prospective Register of Systematic Reviews and published in an open access journal. ${ }^{16}$ The presentation of the findings was guided by the Preferred Reporting Items for Systematic Reviews and Meta-Analyses checklist. ${ }^{17}$

\section{Search strategy}

Due to the extensive number of studies identified within the searched databases, shifts in clinical classifications and TBI definitions during the past 20 years, and the limited empirical evidence regarding the impact of searching and inclusion of earlier works on systematic review findings,${ }^{18}$ our search for relevant articles covered publication period from May 1997 to January 2019 within the following databases:

1. MEDLINE (including Medline in Process and other non-indexed citations, ePubs and Medline Daily).

2. Embase.

3. Cochrane Central Register of Controlled Trials.

4. PsycINFO.

Please see published protocol and online supplementary file 1 for specifics on data searches and MeSH (Medical Subject Headings) terms used. ${ }^{16}$

\section{Inclusion and exclusion criteria}

Studies that were included met the following criteria: (1) focused on comorbidity as it related to our outcome of interest in adults (ie, $\geq 18$ years of age) diagnosed with a TBI on the basis of predefined definitions within the study; (2) comorbidity was detected by any means excluding self-report; (3) reported the proportion of participants without comorbidity and (4) followed participants for any period of time. Studies that fell into either of the following categories were excluded: (1) evaluated children or adolescents (ie, $<18$ years of age), (2) $>50 \%$ of participants had pre-existing TBIs or severe comorbidity at baseline assessment and the subgroup with incident comorbidity could not be extracted independent of preexisting cases. Furthermore, the following study designs/ formats were excluded: letters to editors, reviews without data, case reports or public reports, conference abstracts articles with no primary data, studies that focus on therapeutic interventions and theses.

\section{Data extraction: selection and coding}

Two researchers (CX and SH) independently screened study titles and/or abstracts and reviewed full texts of manuscripts to determine fulfilment of the inclusion criteria. Discrepancies in opinion were resolved through discussion with a third researcher (TM). A previously developed standardised form was used to assess study quality and synthesise study results from the included articles. ${ }^{19}$ Extracted information included the following: (1) study design, (2) study setting, (3) information of the study population and baseline characteristics, (4) attrition rates, (5) details of the definition(s) of TBI and comorbidity, (6) definition of outcome and timing of measurements, (7) the statistical approach used, (8) predictor variables included in the statistical model and (9) information for the assessment of the risk of bias. Two reviewers ( $\mathrm{CX}$ and $\mathrm{SH}$ ) extracted the data independently, and third reviewer (TM) directed the process, reviewed the quality of data extraction, and mediated a resolution in cases of disagreement by performing a separate assessment, and through follow-up discussions with two reviewers.

\section{Risk of bias (quality) assessment}

The quality of each study was evaluated independently by two reviewers (CX and SH) using the Quality in Prognosis Studies tool to assess risk of bias in studies of prognostic factors. ${ }^{20}$ The assessment of each study quality consisted of the following steps: (1) assessment of seven categories of potential bias sources, including study participation, study design, study attrition, prognostic factor, outcome measurements, confounding measurement and account, as well as, analyses; (2) grading the presence of potential biases in each category as 'yes,' 'partly,' 'no,' or 'unsure' and (3) summarising the overall level of potential bias for each study where '++' was assigned when all seven quality criteria were fulfilled (allowing one 'partly' in each bias category); '+' was assigned when four to six criteria were fulfilled; '-' was assigned when fewer than four criteria were fulfilled (ie, at least one 'yes' in each category). A retrospective cohort study design is weaker than a prospective, and therefore, '++' rating (if achieved) was degraded to ' + '. Studies assigned '++' were referred to as 'high-quality studies', 
studies assigned '+' were referred to as 'moderate quality studies" and studies assigned '-' were referred to as 'lowquality studies'. Details on the process of quality assessment are presented in online supplementary tables 1 and 2. Disagreements between the two reviewers were mediated by a third reviewer (TM), who assessed the study's quality independent of the two reviewers and followed up with a discussion.

\section{Data synthesis}

The included studies were synthesised through tabulation and qualitative description. ${ }^{21}$ There was a plan to investigate the pooled effect on our outcome of interest for each group of comorbid disorders (a meta-analytical component of this review), if the data permitted. However, the high heterogeneity among the included studies, concerning study methodology (design-prospective and retrospective cohort), method of assessment of comorbidity, duration of follow-up, etc), population (age, sex, TBI severity, comorbidity type and severity and medication regimen, etc), as well as study settings (acute care, rehabilitation and community) ruled out meta-analysis.

\section{Patient and public involvement}

Patients and the public were not involved in this review.

\section{RESULTS}

The searches yielded a total of 11396 records, from which 9100 records remained after the duplicates were removed. Of the 9100 records, 179 met the criteria for a full-text screen, of which 65 studies were included for the quality assessment. 38 of the studies were of 'low' quality and were excluded. Reasons for study exclusion with specific risk of biases are reported in online supplementary table 2. These studies were penalised because of biases on multiple levels, four or more out of seven criteria of biases. A total of $27^{22-48}$ studies, all of 'moderate' quality, were included for data analysis (figure 1).

\section{Study characteristics}

A summary of the included studies is presented in Table 1, online supplementary tables 3 and 4 . Among the 27 studies, 14 were population based $232526282933-3740414445$ and 13 were clinical studies. ${ }^{22} 2427$ 30-32 $3839424346-48$ Of the 14 population-based studies, all but one used a retrospective cohort design. ${ }^{29}$ Of the 13 clinical studies, four used a prospective cohort methodology 2273943 and nine used a retrospective cohort methodology. ${ }^{24}$ 30-32 384246 With respect to TBI severity, 10 studies included patients of all TBI severities, ${ }^{29} 3233 \quad 35-3739414346$ six studies included moderate and severe TBI cases, ${ }^{24} 3038454748$ and one study included patients with mild TBI. ${ }^{22}$ The

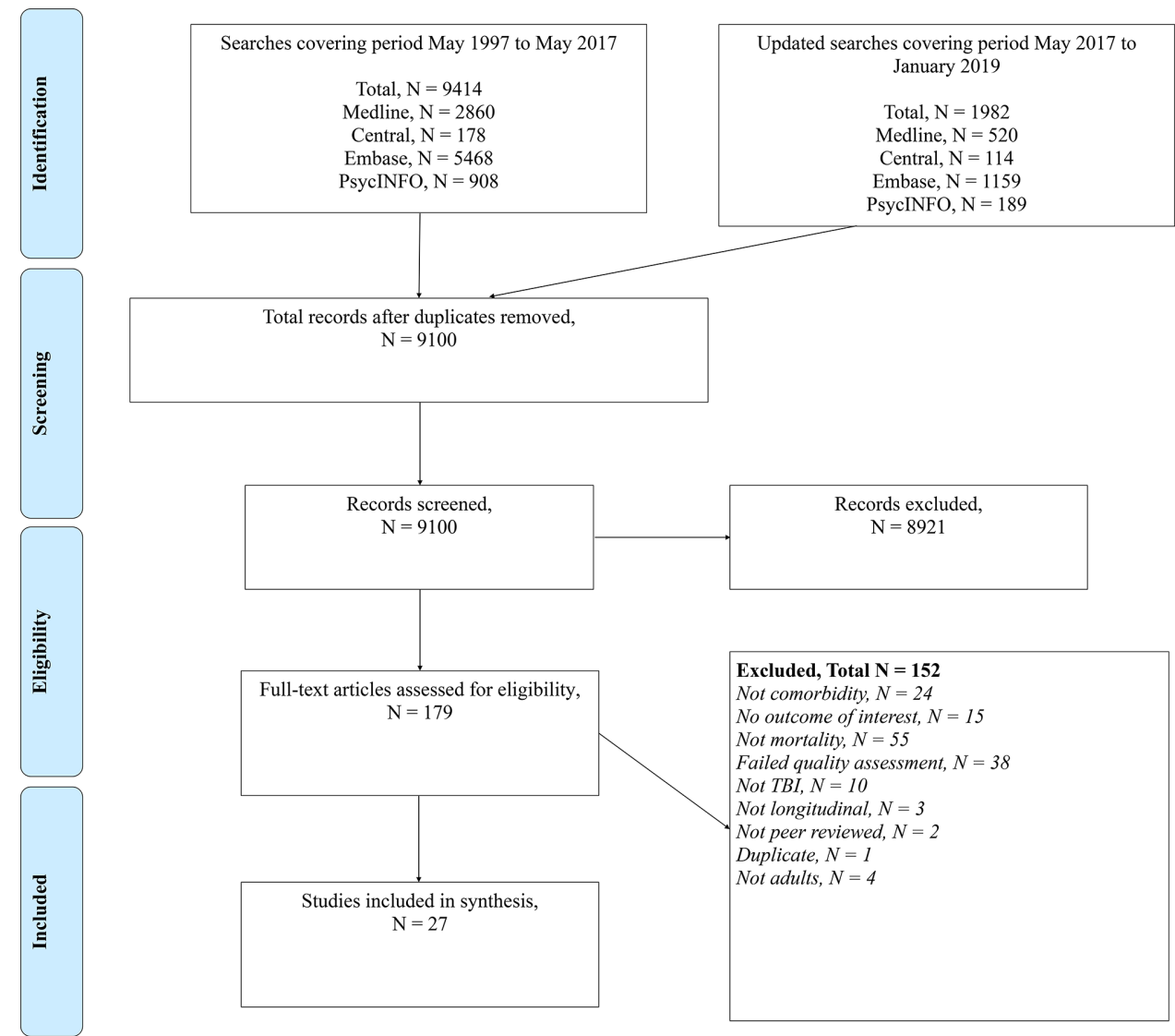

Figure 1 PRISMA diagram. PRISMA, Preferred Reporting Items for Systematic Reviews and Meta-Analyses; TBI, traumatic brain injury. 


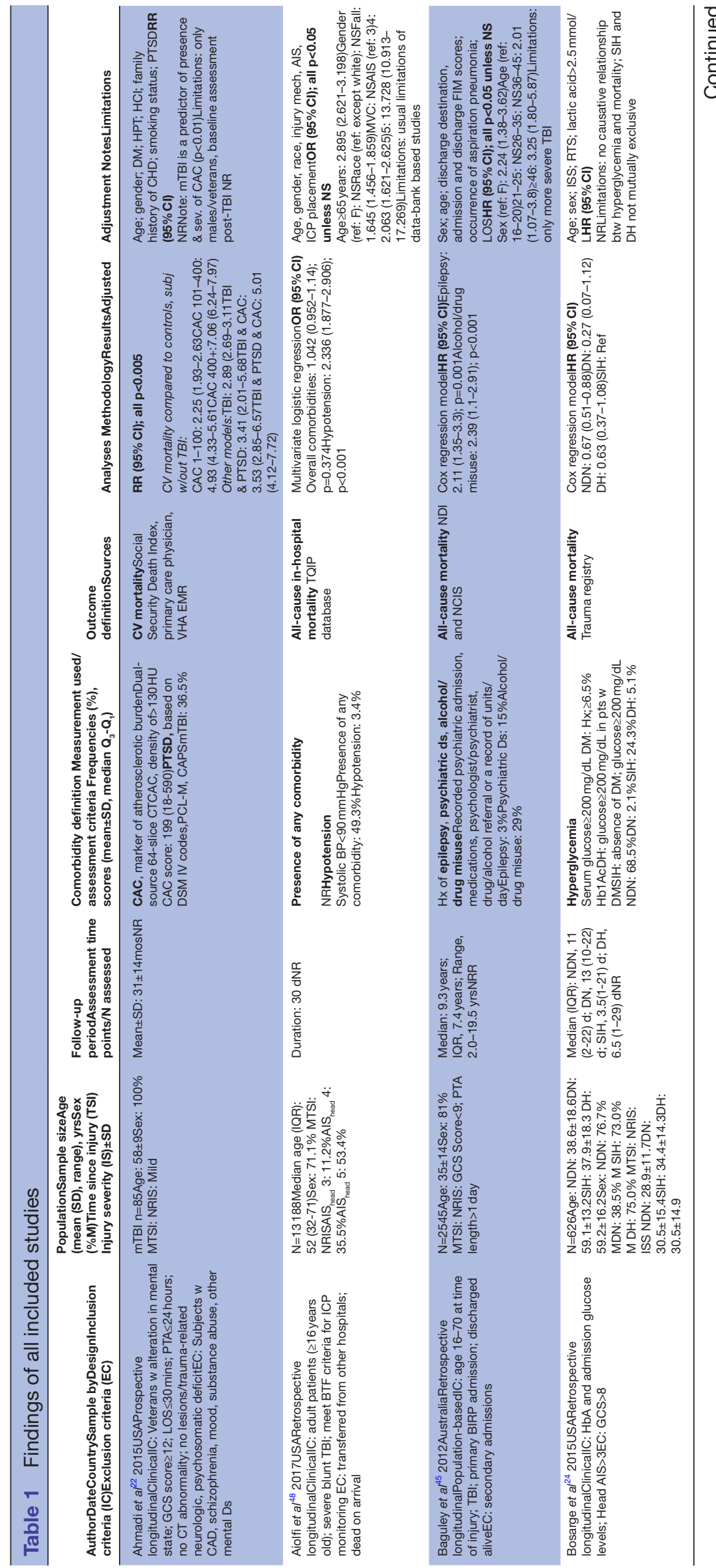




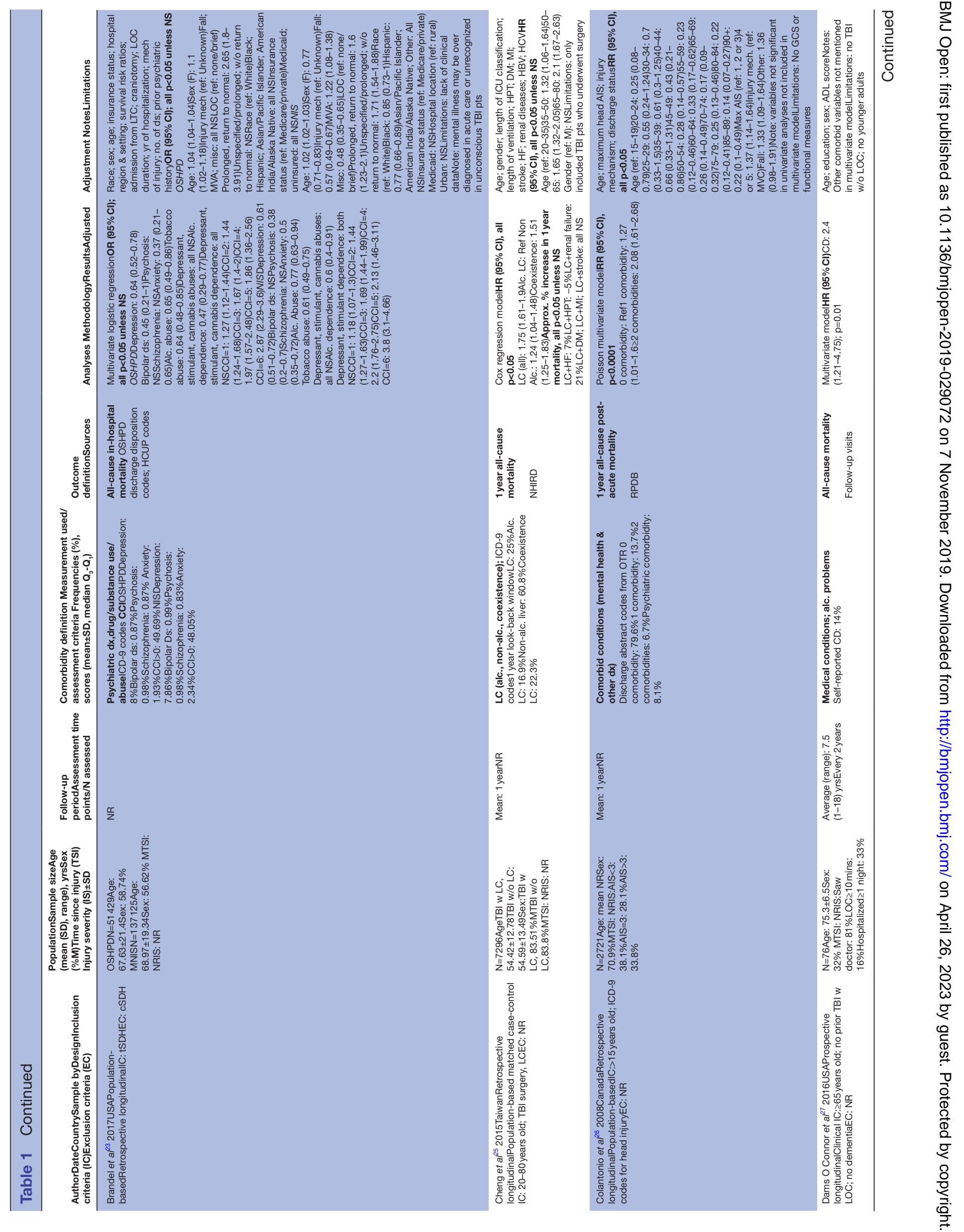



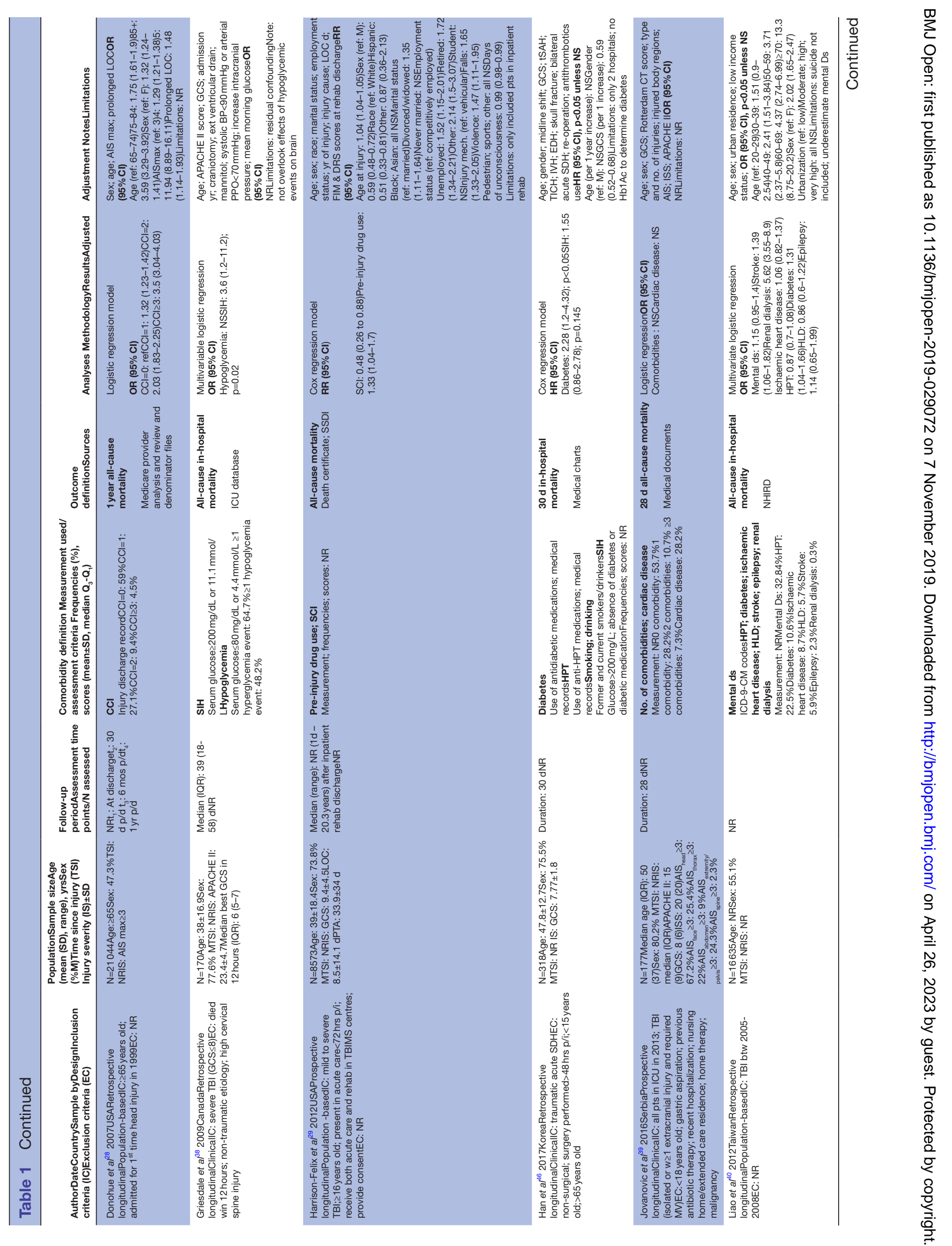


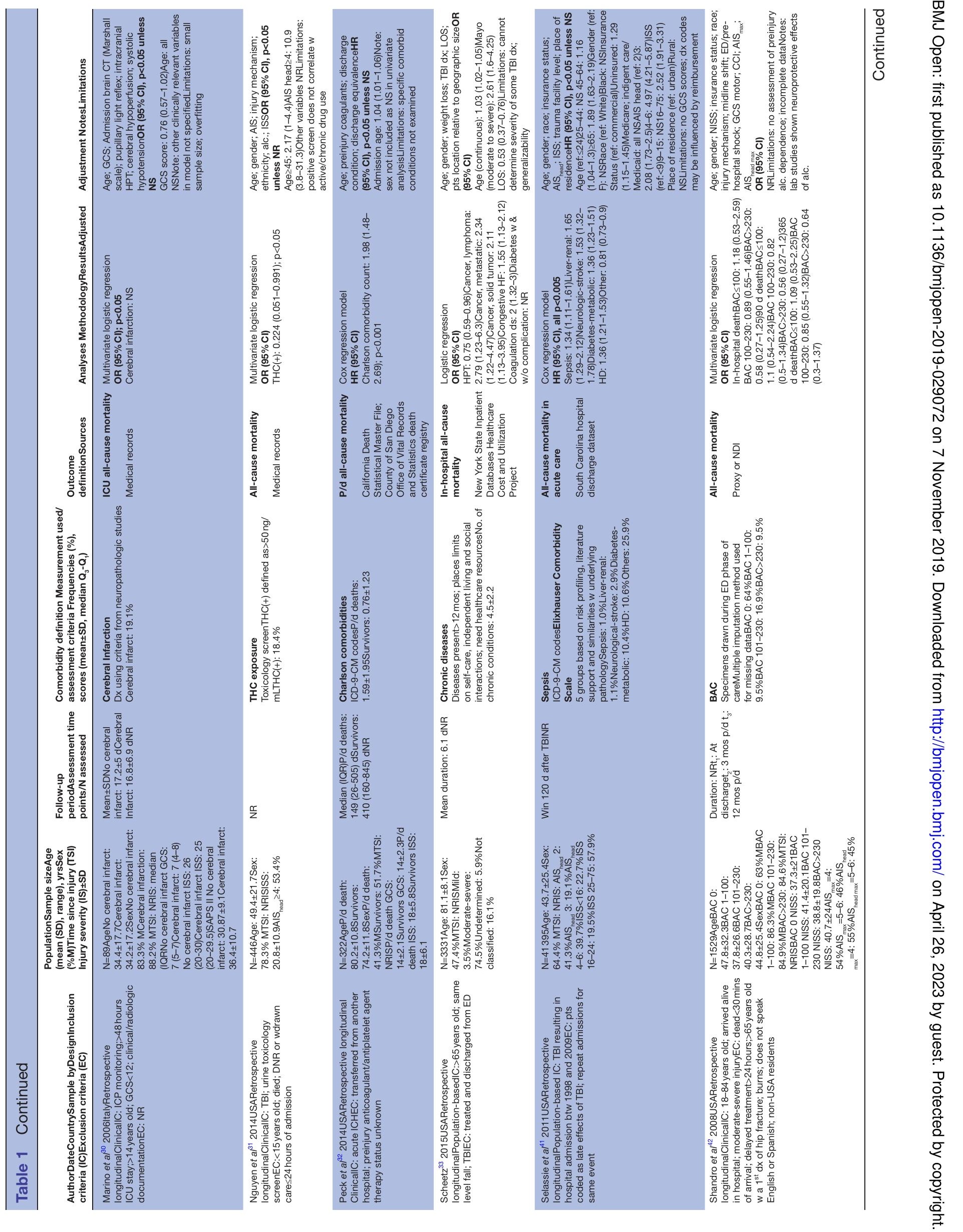




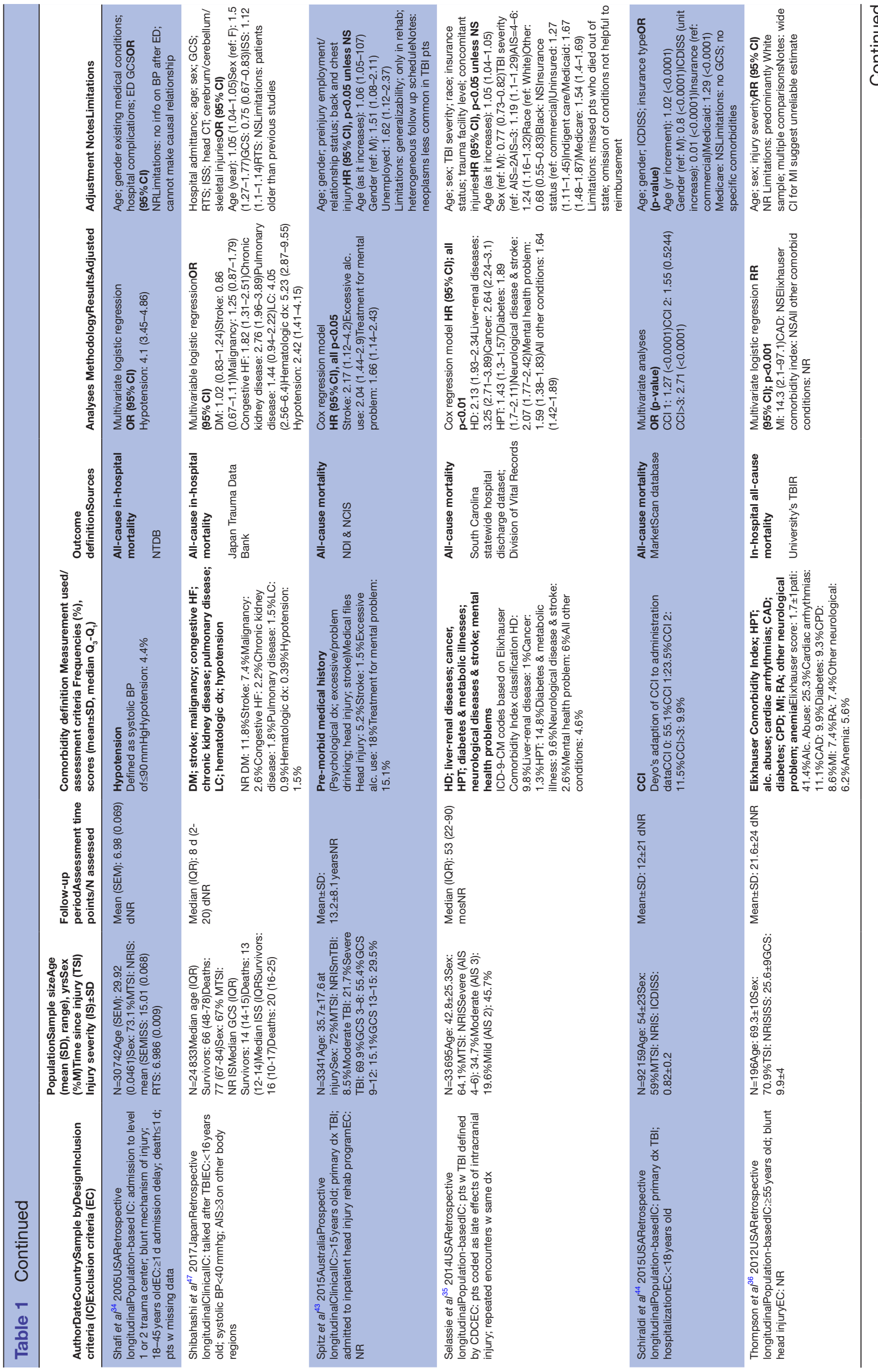



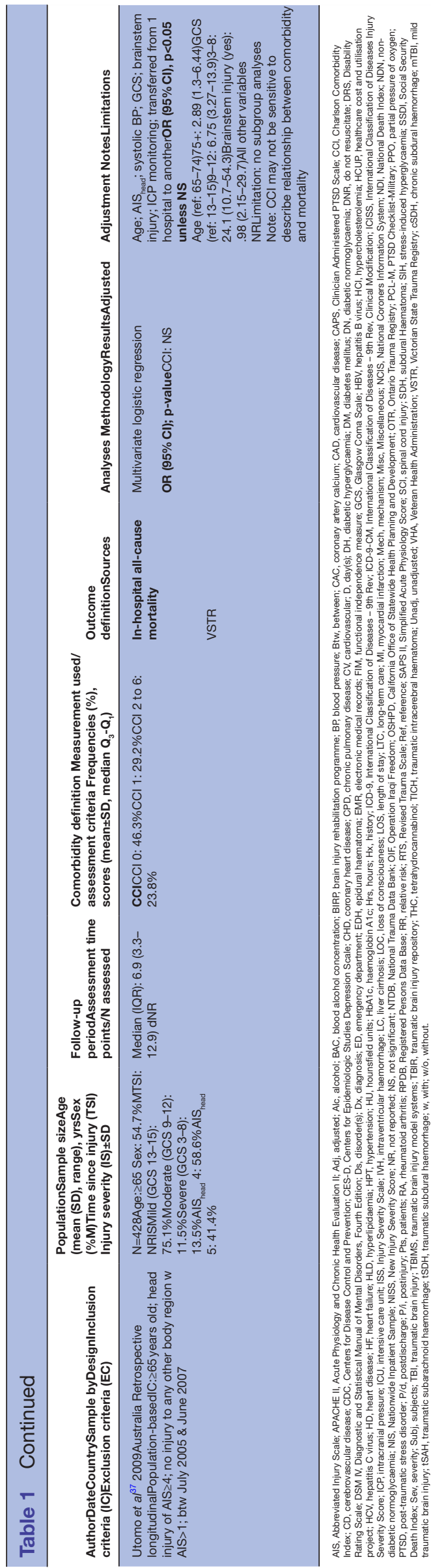

remaining 10 studies did not report information on TBI severity. ${ }^{23} 25-283134404244$

Studied outcomes varied across the included studies. Eleven studies examined long-term ( $>30$ days follow-up) all-cause mortality, ${ }^{25-29} 31323542434515$ studies examined short-term ( $\leq 30$ days follow-up and/or in-hospital) allcause mortality $^{23} 2430333436-414446$ and 1 study examined long-term cardiovascular mortality. ${ }^{22}$

Comorbidity in patients with TBI has been measured using vario

hods including the Charlson Comorbidity Index (CCI), ${ }^{23} 28 \quad 3237 \quad 44 \quad 49$ Elixhauser Comorbidity Index (ECI) ${ }^{35} 364150$ and chronic comorbidity scores determined through pharmacy records. ${ }^{27}$ Two studies reported the association between comorbidity load and mortality by examining the number of comorbid conditions. ${ }^{26}{ }^{39} \mathrm{~A}$ single study examined the relationship between the presence of any comorbidity and mortality. ${ }^{48}$ With respect to comorbidity groups and types, studies examined a wide range of mental and physical conditions. A full list of the comorbidities can be found in table 1 .

\section{Relationship of baseline clinical characteristics and outcome}

Select sociodemographic characteristics and outcome

Among the studies that included age within their final multivariate models, 14 found a significant relationship between increasing age and both short-term and longterm mortality, ${ }^{232528} 2931-333537404143-454748$ two studies did not identify any significant relationship ${ }^{30} 46$ and one study found an increased rate ratio of long-term mortality for the younger age groups compared with the older age groups ( $\geq 50$ years of age). ${ }^{26}$ Effect sizes varied greatly as shown in table 1 . The remaining eight studies did not report their findings on the association between age and mortality. ${ }^{22} 24273436383942$

With respect to sex, seven studies found a reduced risk of both short-term ${ }^{40447}$ and long-term mortality ${ }^{2829354}$ and one study found an increased risk of long-term mortality among females. ${ }^{43}$ Six studies did not find any significant association between sex and mortality rates ${ }^{25} 3135404648$ and a single study demonstrated conflicting findings. ${ }^{23}$ Eight studies did not report their findings on the relationship between sex and mortality. ${ }^{22} 24273334363942$ Finally, several of the included studies also examined and reported the mixed relationships between race, ${ }^{2329} 313541$ marital status, ${ }^{29} 43$ employment status, ${ }^{294}{ }^{43}$ urality $^{23041}$ as well as insurance status ${ }^{23} 354144$ and mortality. Effect sizes varied greatly; details are shown in table 1 .

\section{Select injury characteristics and outcome}

Out of the 12 studies that examined and reported on injury severity in adjusted multivariate analyses, all found a significant association between increasing injury severity and increase in both short-term ${ }^{30} 33374146-48$ and long-term ${ }^{26} 283544$ mortality. ${ }^{31}$ We refer the reader to table 1 for effect sizes reported in individual studies. TBI resulting from falls, ${ }^{26}{ }^{29}$ violence ${ }^{29}$ and all other causes ${ }^{26}$ were found to be significantly associated with increased 
risk of long-term mortality when compared with TBI from motor vehicle collisions. On the contrary, one study did not report any significant findings. ${ }^{31}$

\section{Relationship of comorbidity and outcome Measures used and outcome}

Adjusting for confounders, four of the five studies identified a varying effect size, but all significant association, between CCI and mortality after TBI ${ }^{23} 283244$ (table 1). Among the three studies that used the ECI, ${ }^{35} 3641$ two found significant association between all groups of comorbidities and increased short-term and long-term mortality rates, ${ }^{3541}$ with exception of the 'other' category, which was not significantly associated with short-term post-TBI mortality. ${ }^{41}$ The final study that used the ECI found low sensitivity and specificity for prediction of short-term mortality. ${ }^{36}$

\section{Comorbid condition load and outcome}

Two studies evaluated the relationship between the number of comorbid conditions and mortality after TBI. ${ }^{26}{ }^{39}$ The first study found no significant associations between the number of comorbid conditions and shortterm mortality. ${ }^{39}$ Another study that investigated 1-year mortality found a significant relationship between having one or more comorbidities and long-term mortality. ${ }^{26}$ One study examining the association of the presence of any comorbidity and short-term mortality did not report any significant findings. ${ }^{48}$

\section{Comorbidity type and outcome}

Among comorbidity groups, mental health disorders were the most commonly examined comorbid conditions, with seven studies examining its relationship with post-TBI mortality across varying severities. ${ }^{22} 232629404345$ Effect sizes varied greatly (table 1 ). While one study found significant association between mental health conditions and post-TBI mortality, ${ }^{43}$ three others did not. ${ }^{26} 4045$ Similarly, findings on individual mental health disorders were also mixed.

Five studies examined the role of epilepsy and stroke in predicting mortality post-TBI with varying results. ${ }^{27} 40434547$ Epilepsy was found to be a significant predictor of longterm mortality post severe-TBI mortality ${ }^{45}$ but not shortterm in-hospital morality. ${ }^{40}$ Stroke was found to be significantly associated with an increase in both shortterm in-hospital and long-term mortality post-TBI across severities. ${ }^{27} 4043$ with the exception of one study. ${ }^{47} \mathrm{We}$ refer the reader to table 1 for specifics.

With respect to cardiovascular diseases, the two studies which examined this comorbidity found no significant association with short-term post-TBI mortality ${ }^{39} 40$ while four studies found significant associations between specific heart conditions and increases in both short-term and long-term mortality. ${ }^{22} 333647$ Studies also reported conflicting findings for the role of hypertension in predicting short-term mortality among patients. ${ }^{33} 4046$ All of the three studies that examined low blood pressure (i.e., hypotension) found significant associations with shortterm mortality. ${ }^{344748}$ Of the two studies that examined the association between cancer and short-term mortality, one reported significant findings ${ }^{33}$ while the other did not. ${ }^{47}$ When examining liver-renal disorders, studies found significant associations between liver cirrhosis, renal dialysis and increased long-term and short-term post-TBI mortalities, respectively. ${ }^{25} 4047$ In addition, one study found significantly higher long-term mortality rates among individuals with liver cirrhosis and heart failure, hypertension and/or renal failure compared with those without. ${ }^{25}$ Finally, two studies examining diabetes found significant associations with increased short-term post-TBI mortality. ${ }^{40}{ }^{46}$ while one did not. ${ }^{47}$ Among studies that examined stress-induced high levels of sugar, or glucose, in the blood (i.e., hyperglycaemia), two studies found significant associations with short-term mortality ${ }^{2438}$ while a single study did not ${ }^{46}$ (table 1 ).

In addition to comorbidity groups, several studies examined the association between specific comorbid conditions and clinical signs and mortality. Among the comorbid conditions and co-occurring conditions, hypoglycaemia, ${ }^{38}$ disorders of the blood, ${ }^{47}$ coagulation (i.e., blood clotting) disorders ${ }^{33}$ and sepsis (i.e., systemic inflammatory response to infection $)^{41}$ were significantly associated with increased short-term mortality, and spinal cord injury ${ }^{29}$ was significantly associated with decreased long-term mortality. On the other hand, smoking, ${ }^{46}$ hyperlipidaemia (i.e., harmful cholesterol levels), ${ }^{40}$ alcohol consumption ${ }^{46}$ and cerebral infarction ${ }^{30}$ were not found to be associated with short-term mortality. Similarly, having a previous head injury also did not predict longterm mortality. ${ }^{43}$ Finally, two studies examined the association between alcohol and tetrahydrocannabinol (THC) exposure during injury and mortality postinjury among individuals with unknown TBI severities. While not indicative of any comorbid conditions, these studies found reduced odds of mortality post-TBI for patients who are exposed to THC or alcohol at the time of injury. ${ }^{314}$

\section{DISCUSSION}

Given the large degree of heterogeneity in the characteristics of each study population, the methods used to measure different types of comorbidity, as well as how the outcomes were defined and presented among the 27 included studies, a meta-analysis could not be conducted.

\section{Relationship of baseline clinical characteristics and outcomes Confounding effect}

When examining the relationship between comorbidities and mortality, all studies adjusted their findings by baseline sociodemographic and clinical characteristics of the patients with TBI. Specifically, all studies included age as a variable within their final adjusted models. All but four studies also adjusted their findings by sex and/or gender. Among the studies that did not include sex and/or gender as a confounder in the analyses, two excluded the variable as it was not significant in univariate analyses, ${ }^{2632}$ one excluded the variable as it was neither clinically nor statistically 
significant in bivariate analyses ${ }^{30}$ and one did not provide reason for exclusion. ${ }^{37}$ With respect to other confounders, 17 studies included injury severity $242628303134-3941424446-48$ and seven studies included mechanism of injury 23262939404248 as one of the confounding variables, respectively. Two studies adjusted their findings by the time since injury. ${ }^{29} 38 \mathrm{~A}$ complete list of all the confounders included by each study can be found in table 1 .

\section{Select sociodemographic characteristics and outcome}

In summary, several potential clinical characteristics that predict mortality among patients with TBI had been investigated. Concurrent with previous literature, ${ }^{51}{ }^{52}$ most included studies found age to be significant predictor of mortality. However, given the higher expected death rate among the general older population, one study found the rate ratio to be generally lower for the older age groups ( $\geq 50$ years of age) than that of the younger age groups ( $<50$ years of age) in the TBI population. ${ }^{26}$ As such, it is important to consider the death rates among the general population when examining the influence of TBI and other conditions on mortality. While sex and gender had been known to show an influence on post-TBI recovery, such as functional and cognitive outcomes, ${ }^{53} 54$ findings within the included studies were mixed, which is in line with the current lack of consensus on this topic. Additional demographic variables, such as race, marital status, employment status, rurality and insurance status, were also examined. However, given the limited number of studies and mixed findings in this review, it was not possible to determine the effects of these sociodemographic variables on mortality.

All studies that examined injury severity found increasing severity to be associated with increased short-term and long-term mortality postinjury, which was expected. The relationship between injury mechanism and mortality was also examined. However, studies used varying classifications when determining the risk of mortality from each form of injury mechanism. As such, future work should consider adopting a standardised classification of injury mechanism to enhance consistency and aid comparisons across studies.

\section{Comorbidity measures and outcomes}

As a validated comorbidity, the CCI was the most commonly used comorbidity scale among the included studies. ${ }^{49}$ Patients with TBI across all severities with higher scores on the CCI were found to be at higher risk of both short-term and long-term mortality. However, a study on older adults with TBI and short-term mortality reported contradictory findings. ${ }^{37}$ While the CCI had previously been validated in acutely hospitalised older adult population, ${ }^{55}$ a literature search failed to reveal any validation studies on the TBI population. Hence, these conflicting findings could be attributed to the CCI's limitations in describing the relationship between comorbidity and mortality within this specific population.

The ECI was another commonly used comorbidity scale across studies. However, the findings on the TBI population across all severities were mixed. Unlike the CCI, the ECI explicitly excludes causes of substantial comorbidity in elderly patients, including myocardial infarction and stroke ${ }^{56}$ Hence, it may not capture the comorbidities experienced by older adults with TBI, which would account for the lack of association reported in the study. Therefore, modifications may need to be made to the ECI to enhance its ability to capture the full comorbidity profile of the TBI population, especially among older adults.

\section{Comorbidity load and outcomes}

The absolute number and/or presence of comorbidities had been found to be significantly associated with longterm ${ }^{26}$ but not short-term mortality. ${ }^{39} 48$ This suggested that comorbidities may have varying impact on mortality across the life span of an individual post-TBI. However, given the lack of specification on the types of comorbid conditions included in the comorbidity count, caution should be taken when making inferences on the influence of specific comorbidities on mortality,

\section{Comorbidity type and outcomes \\ Mental health disorders}

Among studies examining mental health disorders, findings remained mixed. A single study found a significant relationship between anxiety, bipolar disorder, psychosis, depression and substance abuse and reduced short-term mortality. ${ }^{23}$ These findings highlighted an important methodology concern when establishing psychiatric diagnoses among the TBI population. Specifically, psychiatric disorders may go unrecognised in patients with TBI who are unconscious at the time of hospitalisation. ${ }^{23}$ Given that these individuals who are conscious postinjury are more likely to survive, there is a potential for the rate of psychiatric diagnoses in those who survive to be bolstered, leading to the observed protective effect of psychiatric disorders on post-TBI mortality. As such, efforts should be taken to reduce the information bias by establishing preinjury psychiatric disorders among the TBI.

In addition, two studies found a significant association between exposures to alcohol and THCand reduced mortality. As these prior exposures are determined at time of injury, they were not indicative of any comorbid substance abuse. Nonetheless, they provide insight on the potential neuroprotective effects of alcohol and THC on the brain at time of injury, which is in line with previous preclinical work. ${ }^{5758}$

\section{Neurological/nervous system disorders}

In line with previous literature, epilepsy had been found to have a significant relationship with increased longterm mortality. However, the same relationship was not observed with respect to short-term mortality, which could be attributed to the chronic nature of the disorder. ${ }^{59}$ As such, it is important for postinjury services to take into consideration the complications that individuals with TBI and epilepsy may encounter throughout their healthcare trajectory. Stroke was found to be significantly associated with increases in both short-term and long-term mortality 
post-TBI across all severities. ${ }^{27} 4043$ with the exception of one study. ${ }^{47}$ Given that majority of the studies found significant associations, assessment for the presence of stroke aetiology at time of TBI is critical to enhance the management and mitigation of adverse outcomes associated with stroke comorbidity.

\section{Cardiovascular disorders}

Among studies that examined cardiac diseases, four studies ${ }^{22} 333647$ observed a significant association with increased mortality. While studies that did not find any associations examined groups of cardiac-related conditions, the four studies that found significant associations examined specific heart diseases and markers such as congestive heart failure, myocardial infarction and coronary artery calcium levels. This suggests that specific cardiac conditions and biomarkers may play a role in influencing adverse outcome post-TBI and future work should focus on identifying these conditions.

\section{Liver-renal disorders}

Three studies examined the association between liver and renal disorders and mortality ${ }^{254047}$ with one having explicitly considered the effect of multimorbidity and liver cirrhosis on short-long-term mortality. This is of importance as the cumulative effect of these diseases may lead to the complication of care for individuals with multiple comorbid conditions. ${ }^{60}$ Nonetheless, while all studies found significant associations between these disorders including liver cirrhosis and renal failure with the need for dialysis and increased long-term and shortterm mortalities, respectively, the study setting was limited to patients in Asia (Japan and Taiwan). Given the different aetiologies of these disorders between societies in the East and West, ${ }^{25}$ there is a need to build on this preliminary evidence through further examinations of these conditions in other study populations and settings.

\section{Hypertension and Hypotension}

While most studies examining hypertension did not find significant associations with mortality, one study found hypertension to be protective of short-term mortality among older adults with severe TBI. ${ }^{33}$ Previous research has found improved survival outcomes post-TBI among patients consuming beta-blockers, a commonly prescribed antihypertensive medication. ${ }^{61}$ Given the chronic nature and increasing prevalence of hypertension with age, ${ }^{62}$ older adults are more likely to be using these medications compared with younger adults. As such, the consumption of beta-blockers may account for the decreased odds of short-term mortality among the hypertensive older adult TBI population found by the studies in our review.

A number of studies had established the relationship between low blood pressure and short-term mortality, ${ }^{34} 4748$ which highlights the importance of assessing the presence of hypotension at time of hospital admission in order to mitigate the potential risk of mortality among the TBI population.
Diabetes mellitus and stress-induced hyperglycaemia

While the association between diabetes mellitus and shortterm mortality had been inconsistent, ${ }^{4046} 47$ most studies examining the effects of stress-induced hyperglycaemia, a marker of oxidative stress and catabolic illnesses, identified a significant association with increased short-term mortality in patients with severe TBI except for a single study, which distinguished between patients with stressinduced hyperglycaemia and diabetes. ${ }^{46}$ While one study has differentiated between stress-induced and diabetic hyperglycaemia, they are not mutually exclusive categories as diabetic hyperglycaemic patients may have some degree of stress response invoking their hyperglycaemia. ${ }^{24}$ As a result, further work to distinguish the various causes of stress-induced hyperglycaemia and their relationship with mortality is warranted.

Other disorders, injury, and symptoms and signs

In addition to the comorbidities examined by the multiple studies above, single studies also found comorbidities including hypoglycaemia, ${ }^{38}$ hematologic disorders, ${ }^{47}$ coagulation disorders ${ }^{33}$ and sepsis $^{41}$ to be significantly associated with increased short-term post-TBI mortality and spinal cord injury ${ }^{29}$ significantly associated with decreased long-term mortality. On the other hand, smoking, ${ }^{46}$ hyperlipidaemia, ${ }^{40}$ alcohol consumption ${ }^{46}$ and cerebral infarction ${ }^{30}$ were not found to be associated with short-term mortality. Similarly, having a previous head injury also did not predict long-term mortality. ${ }^{43}$ However, given the moderate quality of the studies and paucity of supporting evidence, these associations ought to be interpreted with caution.

\section{Risk of bias and study methodology}

The study results depend on the quality of included studies, of which none were high quality based on the risk of bias assessment. Included studies were frequently penalised for being retrospective cohort studies, incomplete reporting when dealing with missing data and statistical analysis. Although most studies performed some form of adjustment for confounders, this was often not described in detail. Most studies did not account for severity of the comorbidity under study, or whether or not the studied comorbidity was adequately controlled by medication, remained untreated or was treatment resistant. In addition, many studies did not control for TBI severity. The lack of consistency in variables included in the modelling process (table 1) and details about independency between included variables (ie, risk factors studied), restricts comparison between the studies.

Closer examination of discrepancies between studies' results revealed a methodological difference between studies that observed a significant and non-significant association. To elaborate, most studies that employed administrative databases did not establish a significant association between psychiatric disorders and mortality ${ }^{27} 40$ while most studies that used medical records did. ${ }^{22} 43$ While administrative data had previously been 
found to agree with medical records for recording of comorbidities, there is a tendency for under-reporting of comorbid conditions in administrative data. ${ }^{63}$ As such, the association between comorbidities and mortality can be potentially masked by the reporting discrepancy. Together, these methodological inconsistencies among studies examined preclude conclusive inferences on the role of comorbidities on post-TBI mortality. Future efforts in this field can focus on performing in-depth examinations of these relationships in order to substantiate evidence, which can inform decision-making and planning of healthcare strategies tailored for patients with TBI with these comorbid conditions.

\section{Limitations}

We acknowledge heterogeneity in the included studies, which demonstrate a great deal of variations in the populations studied, forms and types of comorbidities examined and the mortality outcome. Moreover, various methodologies were employed among studies focusing on similar comorbidities and there were a lack of consideration of the onset of these conditions with respect to TBI. As such, the estimates provided by each study could not be pooled together.

In addition, despite mortality being the most robust and reliable outcome, ${ }^{64}$ the unequal sex and age distribution among the primary studies may have affected the generalisability of the estimates to the TBI population. TBI has been historically considered an injury of younger men and older women. ${ }^{65}$ As such, primary studies focusing on older adults have an over-representation of women and vice versa. While most studies attempted to account for sex and age distributions within their regression modelling, there remains a possibility that comorbid conditions that mainly affect under-represented TBI individuals may not have been captured by the studies.

Most studies focused on multiple types or groups of comorbid conditions. However, the time frame of comorbidity determination was unclear. As such, it was not possible to determine if the conditions were pre-existing or had developed in conjunction with the TBI. Moreover, our assumption was that in the case where statistical significance or the magnitude of an association was not reported, despite the inclusion of a variable in a statistical model, the association was not statistically significant. Thus, the roles of some comorbid conditions that were examined but their relationships were not reported could be underestimated in this review. Furthermore, patients who were treated and who were adherent to treatment for their comorbid conditions may also have exhibited other health behaviours, which could lead to residual confounding.

\section{CONCLUSION}

To the best of our knowledge, we conducted the first systematic review to investigate the relationship between comorbidity, and all-cause mortality in populations with TBI, taking into account sociodemographic and clinical characteristics of persons with TBI. Overall, the evidence supported hypotension as a predictor for short-term mortality, and the evidence about other comorbidities and comorbidity load was mixed. Given the high number of comorbid conditions that were examined by single studies, research is required to further substantiate the evidence and address conflicting findings. In addition, an enhanced set of comorbidity scales that are suited for the TBI population will allow for better risk stratification to guide TBI management and treatment. Finally, given the trend towards big data analysis, future large population-based studies with long follow-up periods, a sufficient number of outcome events, a broad range of population demographic and clinical characteristics, and standardised measures used to define comorbidity (preinjury vs postinjury and severity) are needed to explore further the potential prognostic role of comorbidity in TBI mortality and enable comparisons across TBI populations.

Acknowledgements We gratefully acknowledge the involvement of Jessica Babineau, information specialist at the Toronto Rehabilitation Institute-University Health Network for her help with the literature search.

Authors Contributions Protocol concept and design: TM and AC. Registry PROSPERO: CX. Acquisition of data: TM. Administrative, technical and material support: TM, CX and SH. Statistical analysis approach: TM, VC, ZJH, MS and ME. Screening of articles: CX, SH and TM. Drafting of manuscript: CX. Critical revision of the manuscript for important intellectual content: all authors.

Funding This study was supported through provision of data by the Institute for Clinical Evaluative Sciences (ICES). The opinions, results and conclusions reported in this paper are those of the authors. No endorsement by the ICES is intended or should be inferred. Research reported in this publication was supported by the Eunice Kennedy Shriver National Institute of Child Health and Human Development of the National Institutes of Health under Award Number R21HD089106. Tatyana Mollayeva was funded by the postdoctoral research grant from the Alzheimer's Association (AARF-16-442937). Angela Colantonio was funded by the Canadian Institutes of Health Research (CIHR) Chair in Gender, Work and Health (grant no. CGW-126580).

Disclaimer The content is solely the responsibility of the authors and does not necessarily represent the official views of the National Institutes of Health.

Competing interests None declared.

Patient consent for publication Not required.

Provenance and peer review Not commissioned; externally peer reviewed.

Data availability statement All data relevant to the study are included in the article or uploaded as online supplementary information

Open access This is an open access article distributed in accordance with the Creative Commons Attribution Non Commercial (CC BY-NC 4.0) license, which permits others to distribute, remix, adapt, build upon this work non-commercially, and license their derivative works on different terms, provided the original work is properly cited, appropriate credit is given, any changes made indicated, and the use is non-commercial. See: http://creativecommons.org/licenses/by-nc/4.0/.

ORCID iD

Tatyana Mollayeva http://orcid.org/0000-0002-2787-1848

\section{REFERENCES}

1 Menon DK, Schwab K, Wright DW, et al. Position statement: definition of traumatic brain injury. Arch Phys Med Rehabil 2010;91:1637-40.

2 Maas Al, Menon DK, Adelson PD, et al. Traumatic brain injury: integrated approaches to improve prevention, clinical care, and research. Lancet Neurol 2017;16:987-1048.

3 The Lancet $\mathrm{N}$. The changing landscape of traumatic brain injury research. Lancet Neurol 2012;11.

4 Schwarzbold ML, Rial D, De Bem T, et al. Effects of traumatic brain injury of different severities on emotional, cognitive, and oxidative stress-related parameters in mice. J Neurotrauma 2010;27:1883-93. 
5 Colantonio A, Saverino C, Zagorski B, et al. Hospitalizations and emergency department visits for TBI in Ontario. Can J Neurol Sci 2010;37:783-90.

6 Maas Al, Stocchetti N, Bullock R. Moderate and severe traumatic brain injury in adults. Lancet Neurol 2008;7:728-41.

7 Soo M, Robertson LM, Ali T, et al. Approaches to ascertaining comorbidity information: validation of routine Hospital episode data with clinician-based case note review. BMC Res Notes 2014;7:253.

8 Young JS, Hobbs JG, Bailes JE. The impact of traumatic brain injury on the aging brain. Curr Psychiatry Rep 2016;18:81

9 Feinstein AR. The pre-therapeutic classification of co-morbidity in chronic disease. J Chronic Dis 1970;23:455-68.

10 Chan V, Mollayeva T, Ottenbacher KJ, et al. Clinical profile and comorbidity of traumatic brain injury among younger and older men and women: a brief research notes. BMC Res Notes 2017;10:371.

11 Brooks JC, Shavelle RM, Strauss DJ, et al. Long-Term survival after traumatic brain injury Part II: life expectancy. Arch Phys Med Rehabil 2015;96:1000-5

12. Fu TS, Jing R, McFaull SR, et al. Recent trends in hospitalization and in-hospital mortality associated with traumatic brain injury in Canada: a nationwide, population-based study. J Trauma Acute Care Surg 2015;79:449-55.

13 Masel BE, DeWitt DS. Traumatic brain injury: a disease process, not an event. J Neurotrauma 2010;27:1529-40.

14 Cheng P, Yin P, Ning P, et al. Trends in traumatic brain injury mortality in China, 2006-2013: a population-based longitudinal study. PLoS Med 2017;14:e1002332.

15 Klauber MR, Marshall LF, Barrett-Connor E, et al. Prospective study of patients hospitalized with head injury in San Diego County, 1978. Neurosurgery 1981;9:236-41.

16 Mollayeva T, Xiong C, Hanafy S, et al. Comorbidity and outcomes in traumatic brain injury: protocol for a systematic review on functional status and risk of death. BMJ Open 2017;7:e018626.

17 Moher D, Liberati A, Tetzlaff J, et al. Preferred reporting items for systematic reviews and meta-analyses: the PRISMA statement. PLoS Med 2009;6:e1000097.

18 Hartling L, Featherstone R, Nuspl M, et al. Grey literature in systematic reviews: a cross-sectional study of the contribution of non-English reports, unpublished studies and dissertations to the results of meta-analyses in child-relevant reviews. BMC Med Res Methodol 2017;17:64.

19 Mollayeva T, Kendzerska T, Mollayeva S, et al. A systematic review of fatigue in patients with traumatic brain injury: the course, predictors and consequences. Neurosci Biobehav Rev 2014;47:684-716.

20 Hayden JA, van der Windt DA, Cartwright JL, et al. Assessing bias in studies of prognostic factors. Ann Intern Med 2013;158:280-6.

21 Slavin RE. Best evidence synthesis: an intelligent alternative to meta-analysis. J Clin Epidemiol 1995;48:9-18.10.1016/08954356(94)00097-A

22 Ahmadi N, Hajsadeghi F, Yehuda R, et al. Traumatic brain injury, coronary atherosclerosis and cardiovascular mortality. Brain Injury 2015;29:1635-41.

23 Brandel MG, Hirshman BR, McCutcheon BA, et al. The association between psychiatric comorbidities and outcomes for inpatients with traumatic brain injury. J Neurotrauma 2017;34:1005-16.

24 Bosarge PL, Shoultz TH, Griffin RL, et al. Stress-Induced hyperglycemia is associated with higher mortality in severe traumatic brain injury. J Trauma Acute Care Surg 2015;79:289-94.

25 Cheng C-Y, Ho C-H, Wang C-C, et al. One-Year mortality after traumatic brain injury in liver cirrhosis Patients $-A$ ten-year population-based study. Medicine 2015;94:e1468.

26 Colantonio A, Escobar MD, Chipman M, et al. Predictors of postacute mortality following traumatic brain injury in a seriously injured population. J Trauma 2008;64:876-82.

27 Dams-O'Connor K, Gibbons LE, Landau A, et al. Health problems precede traumatic brain injury in older adults. J Am Geriatr Soc 2016;64:844-8.

28 Donohue JT, Clark DE, DeLorenzo MA. Long-Term survival of Medicare patients with head injury. J Trauma 2007;62:419-23.

29 Harrison-Felix C, Kreider SED, Arango-Lasprilla JC, et al. Life expectancy following rehabilitation: a NIDRR traumatic brain injury model systems study. J Head Trauma Rehabil 2012;27:E69-80.

30 Marino R, Gasparotti R, Pinelli L, et al. Posttraumatic cerebral infarction in patients with moderate or severe head trauma. Neurology 2006;67:1165-71.

31 Nguyen BM, Kim D, Bricker S, et al. Effect of marijuana use on outcomes in traumatic brain injury. Am Surg 2014;80:979-83.

32 Peck KA, Calvo RY, Sise CB, et al. Death after discharge: predictors of mortality in older brain-injured patients. J Trauma Acute Care Surg 2014;77:978-83.
33 Scheetz LJ. Injury patterns, severity and outcomes among older adults who sustained brain injury following a same level fall: a retrospective analysis. Int Emerg Nurs 2015;23:162-7.

34 Shafi S, Gentilello L. Hypotension does not increase mortality in brain-injured patients more than it does in Non-Brain-Injured patients. J Trauma 2005;59:830-5.

35 Selassie AW, Cao Y, Church EC, et al. Accelerated death rate in population-based cohort of persons with traumatic brain injury. $J$ Head Trauma Rehabil 2014;29:E8-19.

36 Thompson HJ, Dikmen S, Temkin N. Prevalence of comorbidity and its association with traumatic brain injury and outcomes in older adults. Res Gerontol Nurs 2012;5:17-24.

37 Utomo WK, Gabbe BJ, Simpson PM, et al. Predictors of in-hospital mortality and 6-month functional outcomes in older adults after moderate to severe traumatic brain injury. Injury 2009;40:973-7.

38 Griesdale DEG, Tremblay M-H, McEwen J, et al. Glucose control and mortality in patients with severe traumatic brain injury. Neurocrit Care 2009;11:311-6.

39 Jovanovic B, Milan Z, Djuric O, et al. Twenty-Eight-Day mortality of blunt traumatic brain injury and Co-Injuries requiring mechanical ventilation. Med Princ Pract 2016;25:435-41.

40 Liao CC, Chiu WT, Yeh CC, et al. Risk and outcomes for traumatic brain injury in patients with mental disorders. J Neurol Neurosurg Psychiatry 2012;83:1186-92.

41 Selassie AW, Fakhry SM, Ford DW. Population-Based study of the risk of in-hospital death after traumatic brain injury: the role of sepsis J Trauma 2011;71:1226-34.

42 Shandro JR, Rivara FP, Wang J, et al. Alcohol and risk of mortality in patients with traumatic brain injury. J Trauma 2009;66:1584-90.

43 Spitz G, Downing MG, McKenzie D, et al. Mortality following traumatic brain injury inpatient rehabilitation. J Neurotrauma 2015;32:1272-80.

44 Schiraldi M, Patil CG, Mukherjee D, et al. Effect of insurance and racial disparities on outcomes in traumatic brain injury. J Neurol Surg A Cent Eur Neurosurg 2015;76:224-32.

45 Baguley IJ, Nott MT, Howle AA, et al. Late mortality after severe traumatic brain injury in New South Wales: a multicentre study. Med $J$ Aust 2011;196:40-5.

$46 \mathrm{Han} \mathrm{M}-\mathrm{H}$, Ryu JI, Kim CH, et al. Radiologic findings and patient factors associated with 30-day mortality after surgical evacuation of subdural hematoma in patients less than 65 years old. $J$ Korean Neurosurg Soc 2017;60:239-49.

47 Shibahashi K, Sugiyama K, Okura Y, et al. Multicenter Retrospective Cohort Study of "Talk and Die" After Traumatic Brain Injury. World Neurosurg 2017;107:82-6.

48 Aiolfi A, Benjamin E, Khor D, et al. Brain trauma Foundation guidelines for intracranial pressure monitoring: compliance and effect on outcome. World J Surg 2017;41:1543-9.

49 Charlson M, Szatrowski TP, Peterson J, et al. Validation of a combined comorbidity index. J Clin Epidemiol 1994;47:1245-51.

50 Elixhauser A, Steiner C, Harris DR, et al. Comorbidity measures for use with administrative data. Med Care 1998;36:8-27.

51 Dhandapani SS, Manju D, Sharma BS, et al. Prognostic significance of age in traumatic brain injury. J Neurosci Rural Pract 2012;3:131-5.

52 Mosenthal AC, Lavery RF, Addis M, et al. Isolated traumatic brain injury: age is an independent predictor of mortality and early outcome. J Trauma 2002;52:907-11.

53 Lavoie S, Sechrist S, Quach N, et al. Depression in men and women one year following traumatic brain injury (TBI): a TBI model systems study. Front Psychol 2017;8:634.

54 Kirkness CJ, Burr RL, Mitchell PH, et al. Is there a sex difference in the course following traumatic brain injury? Biol Res Nurs 2004;5:299-310.

55 Frenkel WJ, Jongerius EJ, Mandjes-van Uitert MJ, et al. Validation of the Charlson comorbidity index in acutely hospitalized elderly adults: a prospective cohort study. J Am Geriatr Soc 2014;62:342-6.

56 Gagne JJ, Glynn RJ, Avorn J, et al. A combined comorbidity score predicted mortality in elderly patients better than existing scores. $J$ Clin Epidemiol 2011;64:749-59.

57 Brennan JH, Bernard S, Cameron PA, et al. Ethanol and isolated traumatic brain injury. Journal of Clinical Neuroscience 2015;22:1375-81.

58 Fernández-Ruiz J, Moro MA, Martínez-Orgado J. Cannabinoids in neurodegenerative disorders and Stroke/Brain trauma: from preclinical models to clinical applications. Neurotherapeutics 2015:12:793-806.

59 Jacoby A, Snape D, Baker G. Epilepsy and social identity: the stigma of a chronic neurological disorder. The Lancet Neurology 2005;4:171-8. 
60 St John PD, Tyas SL, Menec V, et al. Multimorbidity, disability, and mortality in community-dwelling older adults. Can Fam Physician 2014;60:e272-80.

61 Inaba K, Teixeira PGR, David J-S, et al. Beta-Blockers in isolated blunt head injury. J Am Coll Surg 2008;206:432-8.

62 Ong KL, Cheung BMY, Man YB, et al. Prevalence, awareness, treatment, and control of hypertension among United States adults 1999-2004. Hypertension 2007;49:69-75.
63 Quan H, Parsons GA, Ghali WA. Validity of information on comorbidity derived from ICD-9-CCM administrative data. Med Care 2002;40:675-85.

64 Glaab T, Vogelmeier C, Buhl R. Outcome measures in chronic obstructive pulmonary disease (COPD): strengths and limitations. Respir Res 2010;11:79.

65 Thompson HJ, McCormick WC, Kagan SH. Traumatic brain injury in older adults: epidemiology, outcomes, and future implications. J Am Geriatr Soc 2006;54:1590-5. 